\title{
PROTAC the protein
}

\section{By Kai-Jye Lou, Staff Writer}

GlaxoSmithKline plc and Yale University researchers have announced a collaboration to develop a platform that selectively tags disease-associated proteins with an E3 ubiquitin ligase ligand, thus targeting them to a cell's protein degradation machinery. GSK hopes the platform could be a cornerstone for a new Discovery Performance Unit at the pharma, and the partners are aiming to have proof-of-principle results in cell culture by year end.

The platform is based on a chemistry technology called proteolysistargeting chimeric molecules, or PROTACs, which was first reported by Craig Crews at Yale and colleagues at the California Institute of Technology in 2001. ${ }^{1}$ In a pair of recent papers, Crews' team brought the technology closer to therapeutic application by developing small molecule PROTACs. ${ }^{2,3}$

In contrast to typical small molecule inhibitors that block a protein's activity by binding its active site, PROTACs inhibit activity by promoting a protein's degradation via the ubiquitin-proteasome system. The heterodimeric PROTACs do this by forming noncovalent links between the target protein and an E3 ubiquitin ligase, which then marks the protein for degradation by ubiquitinating it.

Crews said PROTACs could make it possible to target intracellular proteins, such as transcription factors and the GTPase K-Ras, that historically have been undruggable with conventional small molecules.

"The structure of the

"We have recently come up with a small molecule E3 ligand, which we are now using as a starting point in the design of molecules that could be linked with targeting moieties against various proteins."

-Craig Crews, Yale University target protein isn't even required, as one just needs to screen molecular libraries for compounds that bind to the target. This liberates one from the limitation of having to develop molecules that target an active site," said Crews, who is a professor of chemistry, pharmacology, and molecular, cellular and developmental biology at Yale.

He said any location on the target protein in which a molecule binds could be exploited by a PROTAC.

The challenge now is to design PROTACs that are more drug-like, which is where GSK comes in, said Crews.

"Our earlier PROTAC molecules were peptidic and large, which could make them difficult to synthesize on a large scale, unstable and difficult to get into cells," he told SciBX. "But we have recently come up with a small molecule E3 ligand, which we are now using as a starting point in the design of molecules that could be linked with targeting moieties against various proteins."

According to Crews, the move from large and peptidic to small molecule was the key transition that caught GSK's attention. He said GSK reached out to the group last year to discuss the potential of developing PROTACs into medicines.

"Some of the findings from the Yale group last year led us to believe that we could make small molecule medicines with the approach," said Kris Famm, who heads GSK's protein degradation effort. "Small molecules are entities that we have many decades of experience with, and we will be able to use our in-house medicinal chemistry expertise and capabilities to generate such PROTACs with more drug-like properties."

Famm said the idea to explore the therapeutic potential of PROTACs followed a call for proposals for new GSK Discovery Performance Units last fall. GSK Discovery Performance Units are small biotech-like groups that focus on specific R\&D projects. These units submit project proposals and seek funding from the pharma's Discovery Investment Board.

"I reached out to the Crews lab to explore the potential to develop these tools into medicines and to see whether this could be a cornerstone for a new GSK Discovery Performance Unit," Famm told SciBX. "This coincided with progress in the Crews lab on making small molecule E3 ubiquitin ligase-binding moieties."

\section{Running interference}

Crews thinks the key competitor for PROTACs is RNAi, which also offers the ability to target proteins regardless of class.

He said PROTACs could have an advantage over RNAi because the latter operates at the level of RNA and thus may have difficulty distinguishing between different conformations of the same protein.

"Because PROTACs work at the level of proteins, they could, for example, be designed to specifically target an oncogenic form of the protein for degradation without also targeting the normal form expressed in healthy cells," Crews told SciBX.

$\mathrm{He}$ added that technical issues make it difficult to deliver and titrate the effect of RNAi-based molecules.

In addition, Crews noted that the mechanism of PROTACs also could give them an edge over existing drugs that are rapidly metabolized and cleared from the body.

"Instead of having to constantly take a particular drug to achieve the necessary level of inhibition for a therapeutic effect, PROTACs reduce the levels of the protein itself so that even when the PROTAC has been cleared, the body will still need to resynthesize the targeted protein to levels that could be harmful," he said.

Famm said PROTACs could have applications across a range of indications in which a protein is expressed at higher levels in diseased tissue than healthy tissue. GSK is not disclosing the specific disease areas and proteins that it plans to target at this time.

"We've established a set of proof-of-principle proteins that we would like to target, and the goal by the end of the year is to show in cell culture that PROTACs could be used to knock down these target proteins," Famm told SciBX. "We will also want to show that the technology is 


\section{ANALYSIS}

generally applicable and prove that we could generate a broad range of PROTACs that are selective, cell permeable and able to exert their intracellular effect. If preclinical proof of principle is achieved for the PROTAC platform, GSK hopes to lead the way in drugging a range of validated disease targets that haven't been possible to effectively target with traditional inhibitors."

Under the collaboration, the GSK Discovery Investment Board and the university will jointly contribute the resources required for the proof-of-principle studies. Yale will be eligible for undisclosed milestones and royalties on each PROTAC that is selected for further development.

GSK has licensed the PROTAC technology from Yale for use for multiple targets. The university retains control of IP related to the small molecule E3 ubiquitin ligase-binding fragment of the PROTACs and its general use. GSK will have exclusive rights to the IP covering whole PROTACs developed under the collaboration.

Lou, K.-J. SciBX 5(20); doi:10.1038/scibx.2012.514

Published online May 17, 2012

\section{REFERENCES}

1. Sakamoto, K.M. et al. Proc. Natl. Acad. Sci. USA 98, 8554-8559 (2001)

2. Buckley, D.L. et al. J. Am. Chem. Soc. 134, 4465-4468 (2012)

3. Neklesa, T.K. et al. Nat. Chem. Biol. 7, 538-543 (2011)

COMPANIES AND INSTITUTIONS MENTIONED

California Institute of Technology, Pasadena, Calif. GlaxoSmithKline plc (LSE:GSK; NYSE:GSK), London, U.K. Yale University, New Haven, Conn. 